\title{
On the problem of the electrified disc
}

\author{
By E. T. Copson. \\ (Received 7th December, 1945. Read 8th December, 1945.)
}

\section{$\S 1 . \quad$ Introduction}

When a perfectly conducting uniform thin circular disc is kept at a potential $V_{0}$ in an external electrostatic field of potential $\Phi$, electric charge is induced on the surface of the disc; the problem is to find the surface-density ${ }^{1} \sigma$ of this induced charge and its potential $V$ so that the total potential $V+\Phi$ has the constant value $V_{0}$ on the surface of the disc. This problem was first discussed by Green ${ }^{2}$ in 1832 , and the solution in the case when there is no external field was deduced by Lord Kelvin ${ }^{3}$ from the known formula for the gravitational potential of an elliptic homoeoid. The problem is still of interest since similar ideas occur in the theory of diffraction by a circular disc and in the theory of the generation of sound waves by a vibrating disc when the wave-length is large compared with the radius of the disc.

The problem can be reduced to that of solving a pair of dual integral equations, due essentially to $H$. Weber ${ }^{4}$. Let us take the centre of the disc as origin, and the axis of the disc as $O z$; in cylindrical coordinates $(\rho, \phi, z)$, the disc is then defined by $z=0$, $\rho<a$. The potential $V$ vanishes at infinity and is equal to $V_{0}-\Phi$ on the surface of the disc. But since $V_{0}-\Phi$ is assumed to be a onevalued continuous function of position on the disc, its value there can be expanded as a sum of terms of the form $f(\rho) \cos n(\phi+a)$, where $n$ is a positive integer or zero. It suffices to consider the case when $V_{0}-\Phi$ reduces to a single term of this form, the general result being obtained by superposition. In this case, $V$ will be of the form $W \cos n(\phi+a)$ where

$$
W=\int_{0}^{\infty} e^{-t i z i} \psi(t) J_{n}(\rho t) d t
$$

1 By $\sigma$ we mean the total surface density on the two faces of the disc.

2Trans. Camb. Phil. Soc., (Nov. 12, 1832), reprinted in Green's Mathematical Papers (London, 1871), 172-183.

${ }^{3}$ Papers on Electricity and Magnetism (Cambridge, 1884), 175-191.

¿ Journal f. Math., 75 (1873), 75-105. 
But this is the potential due to a distribution of electric charge over the plane $z=0$ of surface density $\sigma(\rho) \cos n(\phi+a)$, where

$$
2 \pi \sigma(\rho)=\int_{0}^{\infty} t \psi(t) J_{n}(\rho t) d t
$$

Hence $\psi(t)$ is the solution of the dual integral equations

$$
\begin{aligned}
& \int_{0}^{\infty} \psi(t) J_{n}(\rho t) d t=f(\rho) \quad(0<\rho<a) \\
& \int_{0}^{\infty} t \psi(t) J_{n}(\rho t) d t=0 \quad(\rho>a) .
\end{aligned}
$$

Solutions of these equations in various special cases have been given by $\operatorname{Weber}^{1}\left(n=0, f(\rho)\right.$ constant), Gallop ${ }^{2}\left(n=0, f(\rho)=J_{0}(c \rho)\right)$, Basset $^{3}\left(n=1, f(\rho)=J_{1}(c \rho)\right)$, and Macdonald ${ }^{4}$ ( $n$ arbitrary $\left.f(\rho)=J_{n}(c \rho)\right)$. The solution when $f(\rho)$ is an arbitrary function was first given by Beltrami ${ }^{5}$ for the case $n=0$ and by L. V. King ${ }^{6}$ for any integral value of $n$. In all these cases the analysis is difficult and requires considerable insight into the properties of the discontinuous integrals of Weber and Schafheitlin?. Quite recently Titchmarsh ${ }^{8}$ has given a more direct solution of the dual integral equations by means of the theory of Mellin transforms.

Although the analysis is difficult, the final results are simple, and this suggests that there should be a simpler solution. In the present note it is shown that this is the case, provided that one does not start from the potential function (1), which is too general for the purpose. When one starts with a more suitable form of potential function, one obtains a single integral equation which involves a repeated integral, and this can be solved by two applications of the known solution of Abel's integral equation.

\footnotetext{
$1 \mathrm{H}$. Weber, loc. cit.

2 Quarterly Journal of Mathematics, 21 (1886), 229-256.

${ }^{3}$ Proc. Camb. Phil. Soc., 5 (1886), 425.443.

4 Proc. London Math. Soc. (1), 26 (1895), 257-260.

5 Bologna Memorie (4), 2 (1880), 461-505.

- Proc. Roy. Soc. (A), 153 (1936), 1-16.

'Cf. Watson, Theory of Bessel Functions (Cimbridge, 1944), 398-406.

$\checkmark$ The Theory of Fourier Integrals (Oxford, 1937), 334-339.
} 


\section{§2. Three Lemmas}

Lemma 1. If $n$ is a positive integer or zero and if $a$ and $b$ are positive

$$
\int_{0}^{2 \pi} \frac{e^{i n \phi} d \phi}{\sqrt{ }\left\{a^{2}+b^{2}-2 a b \cos \phi\right\}}=\frac{4}{a^{n} b^{n}} \int_{0}^{M i n(a, b)} \frac{t^{2 n} d t}{\sqrt{ }\left\{\left(a^{2}-t^{2}\right)\left(b^{2}-t^{2}\right)\right\}}
$$

the two square roots being positive.

On account of the symmetry and homogeneity of the former integral in $a$ and $b$, we may assume that $b=1$ and $0<a<1$. Putting $z=e^{i \phi}$, we have

$$
\left.\int_{0}^{2 \pi} \overline{\sqrt{ }\{a-i}+\frac{e^{i n \phi} d \phi}{1-2 a} \cos \bar{\phi}\right\}=\frac{1}{i} \int_{C} \frac{z^{n} d z}{\sqrt{ }\{z(z-a)(1-a z)\}}
$$

where $C$ is the circle $|z|=1$ and the branch of $\sqrt{ }\{z(z-a)(1-a z)\}$ is chosen which has the value $1-a$ when $z=1$. The integrand is regular in the $z$-plane supposed cut along the real axis from 0 to $a$ and from $1 / a$ to infinity. The value of the integral is unaltered if the path $C$ is deformed into a loop surrounding the cut from 0.to $a$. Making the loop coincide with the two sides of the cut, we obtain

$$
\begin{aligned}
\int_{0}^{2 \pi} \frac{e^{i n \phi} d \phi}{\sqrt{ }\left\{a^{2}+1-2 a \cos \phi\right\}} & =2 \int_{0}^{a} \frac{r^{n} d r}{\sqrt{\{r(a-r)}(1-a r)\}} \\
& =\frac{4}{a^{n}} \int_{0}^{a} \frac{t^{2 n} d t}{\sqrt{ }\left\{\left(a^{2}-t^{2}\right)\left(1-t^{2}\right)\right\}}
\end{aligned}
$$

where $t^{2}=a r . \quad$ This completes the proof of Lemma 1 .

Lemma 2. Let $f(x)$ and $f^{\prime}(x)$ be continuous in $0 \leqq x \leqq a$. Then the solution of the integral equation

is

$$
f(x)=\int_{0}^{x} \frac{g(t)}{\sqrt{ }\left(x^{2}-t^{2}\right)} d t \quad(0<x<a)
$$

$$
g(x)=\frac{2}{\pi} f(0)+\frac{2}{\pi} \int_{0}^{x} \frac{x f^{\prime}(t)}{\sqrt{ }\left(x^{2}-t^{2}\right)} d t \equiv \frac{2}{\pi} \frac{d}{d x} \int_{0}^{x} \frac{t f(t)}{\sqrt{\left(x^{2}-t^{2}\right)}} d t
$$

Lemma 3. Let $f(x)$ and $f^{\prime}(x)$ be continuous in $c \leqq x \leqq a$. Then the solution of the integral equation

is

$$
f(x)=\int_{x}^{a} \frac{g(t)}{\sqrt{\left(t^{2}-x^{2}\right)}} d t \quad(c<x<a)
$$

$g(x)=\frac{2}{\pi} f(a) \frac{x}{\sqrt{ }\left(a^{2}-x^{2}\right)}-\frac{2}{\pi} \int_{x}^{c} \frac{x f^{\prime}(t)}{\sqrt{\left(t^{2}-x^{2}\right)}} d t \equiv-\frac{2}{\pi} \frac{d}{d x} \int_{x}^{a} \frac{t f(t)}{\sqrt{ }\left(t^{2}-x^{2}\right)} d t$. 
Lemmas 2 and 3 are trivial transformations of the well-known solution of Abel's integral equation under conditions which are sufficient for our present purpose. The transformation $t=x \sin \theta$ turns the integral equation of Lemma 2 into Schlömilch's integral equation'.

§3. The solution of the problem of the electrified disc

Theorem. Let the potential on the surface of the circular disc $z=0$, $0<\rho<a$ be $f(\rho) \cos n(\phi+a)$, where a is a constant, $n$ is a positive integer or zero and $f(\rho)$ is continuously differentiable in $0 \leqq \rho \leqq a$.

Let the function

$$
S(\rho)=\underset{2 \pi \rho^{2 n}}{1} \frac{d}{d \rho} \int_{0}^{\rho} \frac{t^{n+1} f(t)}{\sqrt{ }\left(\rho^{2}-t^{2}\right)} d t
$$

be continuously differentiable in $\epsilon \leqq \rho \leqq a$ for any positive value of $\epsilon(<a)$.

Then the surface-density of electric charge on the surface of the disc is $\sigma(\rho) \cos n(\phi+a)$, where

$$
\sigma(\rho)=-\frac{2}{\pi} \rho^{n-1} \frac{d}{d \rho} \int_{\rho}^{a} \frac{t S(t)}{\sqrt{ }\left(t^{2}-\rho^{2}\right)} d t .
$$

The potential at the point of cylindrical coordinates $(\rho, \phi, z)$ due to a distribution of electric charge of surface-density $\sigma(\rho, \phi)$ on the disc is

$$
V=\int_{0}^{a} \int_{0}^{2 \pi} \sigma(u, \theta) \frac{u}{r} d \theta d u
$$

where $r$ is the distance from $(\rho, \phi, z)$ to the point $(u, \theta, 0)$ of the disc. We have to choose $\sigma$ so that $V=f(\rho) \cos n(\phi+a)$ when $z=0$. But if we expand $\sigma(\rho, \phi)$ as a Fourier series in $\phi$ of period $2 \pi$, we see at once that it must reduce to one term, $\sigma(\rho) \cos n(\phi+a)$ say, where $\sigma(\rho)$ has to be chosen so that

$\int_{0}^{a} \sigma(u) u \int_{0}^{2 \pi}-\sqrt{\sqrt{ }\left\{\rho^{2}+u^{2}-2 \rho u \cos (\theta-\phi)\right\}} d \theta d u=f(\rho) \cos n(\phi+a)(4)$

when $0<\rho<a$.

By Lemma 1, we have

$\int_{0}^{2 \pi} \frac{\cos n(\theta+a) d \theta}{\sqrt{ }\left\{\rho^{2}+u^{2}-2 \rho u \cos (\theta-\phi)\right\}}=\frac{4 \cos n(\phi+a)}{\rho^{n} u^{n}} \int_{0}^{\operatorname{Min}(\rho, u)} \frac{t^{2 n} d t}{\sqrt{ }\left\{\left(u^{2}-t^{2}\right)\left(\rho^{2}-t^{2}\right)\right\}}$

I Of. Whittaker and Watson, Modern Analysis (Cambridge, 1920), p. 229. 
Hence equation (4) becomes

$$
\begin{aligned}
\frac{4}{\rho^{n}}\left\{\int_{0}^{\rho} \sigma(u) u^{1-n} \int_{0}^{u}\right. & \frac{t^{2 n} d t}{\sqrt{\left\{\left(u^{2}-t^{2}\right)\left(\rho^{2}-t^{2}\right)\right\}}} d u \\
& \left.+\int_{\rho}^{a} \sigma(u) u^{1-n} \int_{0}^{\rho} \frac{t^{2 n} d t}{\sqrt{ }\left\{\left(u^{2}-t^{2}\right)\left(\rho^{2}-t^{2}\right)\right\}} d u\right\}=f(\rho) .
\end{aligned}
$$

Inverting the order of integration, we obtain

$$
\frac{1}{4} \rho^{n} f(\rho)=\int_{0}^{\rho} \frac{t^{2 n}}{\sqrt{ }\left(\rho^{2}-t^{2}\right)} \int_{t}^{a} \frac{\sigma(u) u^{1-n}}{\sqrt{\left(u^{2}-t^{2}\right)}} d t \quad(0<\rho<a) .
$$

To solve the repeated integral equation (5), we substitute

$$
S(\rho)=\int_{\rho}^{a} \frac{\sigma(u) u^{1-n} d u}{\sqrt{\left(u^{2}-\rho^{2}\right)}} \quad(0<\rho<a)
$$

and obtain

$$
\frac{1}{4} \rho^{n} f(\rho)=\int_{0}^{\rho} \frac{t^{2 n} S(t) d t}{\sqrt{ }\left(\rho^{2}-t^{9}\right)} \quad(0<\rho<a) .
$$

But since $f(\rho)$ and $f^{\prime}(\rho)$ are continuous, we may apply Lemma 2 to (7). This gives

$$
S(\rho)=\frac{1}{2 \pi \rho^{2 n}} \frac{d}{d \rho} \int_{0}^{\rho} \frac{t^{n+1} f(t) d t}{\sqrt{\left(\rho^{2}-t^{2}\right)}}, \quad(0<\rho<a) .
$$

By hypothesis, this function and its first derivative are continuous in $\epsilon \leqq \rho \leqq a$, for any positive value of $\epsilon(<a)$. Hence by Lemma 3,

which solves the problem.

$$
\sigma(\rho)=-\frac{2}{\pi} \rho^{n-1} \frac{d}{d \rho} \int_{\rho}^{a} \frac{t S(t) d t}{\sqrt{\left(t^{2}-\rho^{2}\right)}},
$$

\section{\$4. Some special solutions}

The simplicity of the present method of solution of the problem of the electrified dise is well illustrated by applying it to the special cases mentioned in $\S 1$. That the conditions of the theorem apply in each case is easily verified.

(i) The disc is raised to unit potential with no external field.

Here $n=0$ and $f(\rho)=1$. Hence we have

$$
\begin{aligned}
& S(\rho)=\frac{1}{2 \pi} \frac{d}{d \rho} \int_{0}^{\rho} \frac{t d t}{\sqrt{\left(\rho^{2}-t^{2}\right)}}=\frac{1}{2 \pi}, \\
& \sigma(\rho)=-\frac{1}{\pi^{2} \rho} \frac{d}{d \rho} \int_{\rho}^{a} \frac{t d t}{\sqrt{\left(t^{2}-\rho^{2}\right)}}=\frac{1}{\pi^{2} \sqrt{\left(a^{2}-\rho^{2}\right)}}
\end{aligned}
$$

in agreement with the result of Green, Kelvin and Weber. 
(ii) The disc is earthed in an external uniform field parallel to its surface.

If we take the external field strength to be unity, we have $V_{0}=0, \Phi=\rho \cos \phi$, so that $n=1, f(\rho)=\rho$. Hence

$$
\begin{aligned}
& S(\rho)=\frac{1}{2 \pi \rho^{2}} \frac{d}{d \rho} \int_{0}^{\rho} \frac{t^{3} d t}{\sqrt{ }\left(\rho^{2}-t^{2}\right)}=\frac{1}{\pi} \\
& \sigma(\rho)=-\frac{2}{3 \pi^{2}} \frac{d}{d \rho} \int_{\rho}^{a} \frac{t d t}{\sqrt{\left(t^{2}-\rho^{2}\right)}}=\frac{2 \rho}{\pi^{2} \sqrt{ }\left(a^{2}-\rho^{2}\right)} .
\end{aligned}
$$

(iii) Gallop's problem.

In this case $n=0$ and $f(\rho)=J_{0}(c \rho)$. We easily show that

$$
\begin{aligned}
& S(\rho)=\frac{1}{2 \pi} \frac{d}{d \rho} \int_{0}^{\rho} \frac{t J_{0}(c t)}{\sqrt{ }\left(\rho^{2}-t^{2}\right)} d t=\frac{1}{2 \pi} \frac{d}{d \rho}\left(\frac{\sin c \rho}{c}\right)=\frac{\cos c \rho}{2 \pi}, \\
& \sigma(\rho)=-\frac{1}{\pi^{2} \rho} \frac{d}{d \rho} \int_{\rho}^{a} \frac{t \cos c t}{\sqrt{\left(t^{2}-\rho^{2}\right)}} d t \\
& =\frac{\cos c a}{\pi^{2} \sqrt{\left(a^{2}-\rho^{2}\right)}}+\frac{1}{\pi^{2}} \int_{\rho}^{a} \frac{c \sin c t}{\sqrt{ }\left(t^{2}-\rho^{2}\right)} d t \\
& =\frac{1}{\pi^{2}} \int_{\rho}^{\infty} \frac{c \sin c t}{\sqrt{ }\left(t^{2}-\rho^{2}\right)} d t+\frac{\cos c a}{\pi^{2} \sqrt{\left(a^{2}-\rho^{2}\right)}}-\frac{1}{\pi^{2}} \int_{a}^{\infty} \frac{c \sin c t}{\sqrt{ }\left(t^{2}-\rho^{2}\right)} d t \\
& \doteq \frac{c}{2 \pi} J_{0}(c \rho)+\frac{1}{\pi^{2}} \int_{a}^{\infty} \frac{t \cos c t}{\left(t^{2}-\rho^{2}\right)^{3 / 2}} d t
\end{aligned}
$$

after using Mehler's formula ${ }^{1}$ for $J_{0}(x)$.

(iv) Macdonald's problem.

In this oase $n$ is an arbitrary positive integer and $f(\rho)=J_{n}(c \rho)$. The expressions for $S(\rho)$ and $\sigma(\rho)$ then reduce to

$$
\begin{gathered}
S(\rho)=\frac{c^{n}}{2} \sqrt{\sqrt{(2 \pi)}} \frac{J_{n-\frac{1}{2}}(c \rho)}{(c \rho)^{n-\frac{1}{2}}} \\
\sigma(\rho)=\frac{c}{2 \pi} J_{n}(c \rho)+\frac{c^{n} \rho^{n}}{\sqrt{ }\left(2 \pi^{3}\right)} \int_{a}^{\infty} \frac{J_{n-\frac{1}{2}}(c t)}{(c t)^{n-\frac{1}{2}}} \frac{t d t}{\left(t^{2}-\rho^{2}\right)^{3 / 2}} .
\end{gathered}
$$

${ }^{3}$ Cf. Watson, loc. cit., $180(14)$

Uxiversity College, University of St Andrews, DONDEF. 